\title{
Integrated-optic voltage transducer for high-voltage applications
}

\author{
Patrick P. Chavez ${ }^{a}$, Nicolas A. F. Jaeger ${ }^{a}$, Farnoosh Rahmatian $^{b}$, and Chris Yakymyshyn ${ }^{c}$ \\ ${ }^{a}$ University of British Columbia, Department of Electrical and Computer Engineering, 2356 Main Mall, \\ Vancouver, British Columbia, Canada V6T 124 \\ ${ }^{b}$ NxtPhase Corporation, 3040 East Broadway, \\ Vancouver, BC V5M 1 Z4
}

\author{
${ }^{c}$ Montana State University, Department of Electrical and Computer Engineering \\ 610 Cobleigh Hall, Bozeman, Montana, USA 59717
}

\begin{abstract}
This paper describes a novel voltage transducer. Its design is based on a mathematical procedure that enables a small number of strategically positioned electric field sensors to accurately measure the voltage. The voltage transducer takes advantage of existing compact, non-intrusive optical electric field sensor technology, specifically, the integrated-optic Pockels cell (IOPC), but is not limited to optical technology. The key advantage of this voltage transducer over other existing optics-based voltage transducer technologies is that it does not require any customized electrode structures and/or special insulation. A highvoltage integrated-optic voltage transducer has been used to obtain measurements with metering class accuracies.
\end{abstract}

Keywords: high-voltage, transducer, sensor, electric field, measurement, optics, integrated-optics, numerical integration

\section{INTRODUCTION}

In recent years, electro-optic devices for accurately measuring voltage in high-voltage environments have been developed ${ }^{1,2,3,4,5}$. Compared to conventional technologies, e.g., inductive or capacitive voltage transformers, optical technology offers wider bandwidth, larger dynamic range, immunity to electro-magnetic interference, electrical isolation, lighter weight, and smaller size. As a result, optical voltage sensing technology is poised to replace the conventional technologies in the near future.

Generally, existing optical voltage sensors use bulk-optic Pockels cells surrounded by special electrode structures and/or special insulating gases, such as $\mathrm{SF}_{6}^{1,2,3,4}$, leading to significant complexity in mechanical design and construction. Also, $\mathrm{SF}_{6}$ is not as popular as it once was for environmental reasons.

Here, a new type of optical voltage transducer (VT) design that does not rely on special electrode structures or special insulation and that is synergistic with compact, immersion-type integrated-optic Pockels cell (IOPC) technology ${ }^{5}$ is introduced. This integrated-optic voltage transducer (IOVT) uses very few electric field sensors, which make point-like electric field measurements, to approximately integrate the field, obtaining the voltage between two electrodes. To do this the IOPCs must be located at specific positions and assigned specific weights. This allows the design of the IOVT to be very flexible.

In the following sections, the relationship between the voltage and the electric field, for the approach used here, is briefly outlined, i.e., numerically integrating the electric field by sampling it and weighting the sample values, is explained. This is followed by a specific example of a highly accurate IOVT that uses only three IOPCs and an off-the-shelf post insulator.

\section{VOLTAGE AND ELECTRIC FIELD}

In order to use electric field sensors to measure the voltage on a conductor, e.g., on a particular phase of a three-phase transmission line, the relationship between the measured electric field and the voltage must be understood. It is well-known 
that any electric field distribution depends on the sources of electrical charge that generate it and on the media surrounding them $^{6}$. In the case of a high-voltage substation, the electric field at any point depends on the voltages on all the source conductors and on the layout and physical condition of the substation, e.g., the positions, sizes, and shapes of the buses and equipment as well as the weather conditions. In other words there does not exist a fixed one-to-one relationship between the electric field measured at a point and the voltage on a conductor if the voltages on other nearby conductors and the geometry and/or the dielectric properties of the surroundings are changing.

As an example, the behavior of the electric field inside a post insulator, as the external geometry changes, is investigated. The post insulator consists of an off-the-shelf standoff, a metallic stand, and a metal cap. The standoff is comprised of rubber sheds, inner and outer fiberglass tubes, and flanges on both ends. The diameter of the standoff is about $60 \mathrm{~cm}$ and its height is about $2 \mathrm{~m}$, characteristic of a $230 \mathrm{kV}$ class post insulator. In addition, a corona ring surrounds the top flange protecting it from excessive field stresses. Figure 1(a) shows this post insulator sitting on a ground plane. Figure 1(b) shows the same configuration as Fig. 1(a) with an additional semi-infinite vertical ground plane $1.5 \mathrm{~m}$ away from the axis of the post. In practice, placing large objects, particularly ones that are conducting, this close to a high-voltage conductor is not allowed for safety reasons. Thus, the nearby vertical ground plane represents an overly extreme case of an external influence. Using finite element modeling ${ }^{7,8}$, the electric field distribution is computed for $100 \mathrm{kV}$ applied across the standoff, and a plot of the axial component (the $x$-component) of the electric field along the axis of the standoff from the bottom electrode to the top electrode ( $a$ to $b$ ) for both configurations is shown in Fig. 2. With the vertical ground plane present, the magnitude of the axial field, along the $x$-axis, is different from that obtained when the vertical ground plane is absent.

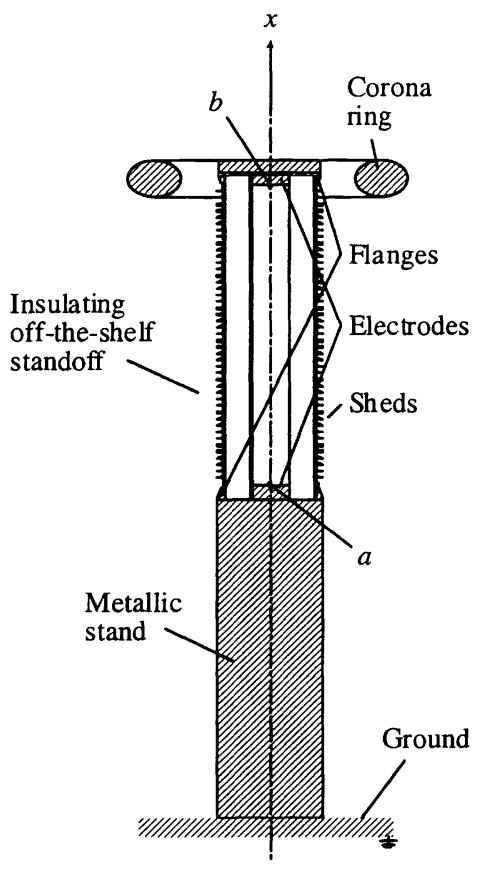

(a)

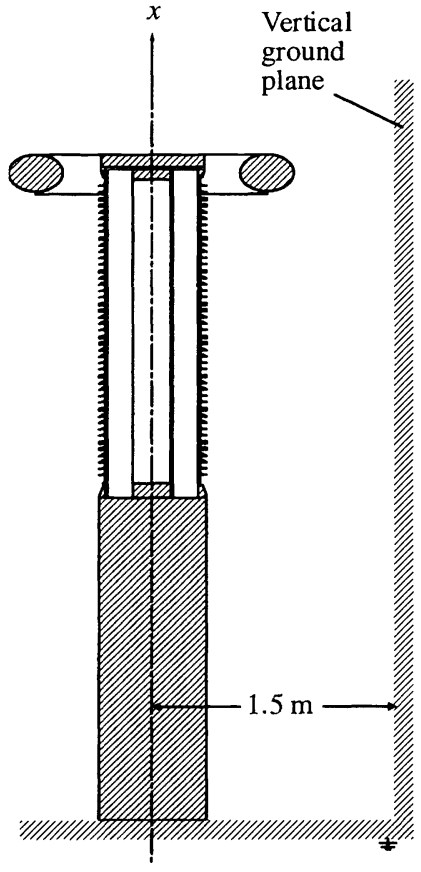

(b)

Figure 1. (a) A post insulator and (b) the same post insulator with a vertical ground plane $1.5 \mathrm{~m}$ away.

As was shown in Fig. 2, the electric field along the $x$-axis changed as a result of the change in the geometry, i.e., when the ground plane was introduced. If a one-to-one relationship between the electric field measured at a particular point and the voltage difference between two conductors is desired, then the geometry of the region surrounding the conductors must be fixed. This approach has been used in optical VTs in the past ${ }^{1,2,3}$. For example, Christensen ${ }^{3}$ enclosed a grounded electrode with a surrounding high-voltage electrode to obtain the fixed relationship and used a bulk-optic Pockels cell to measure the field. 


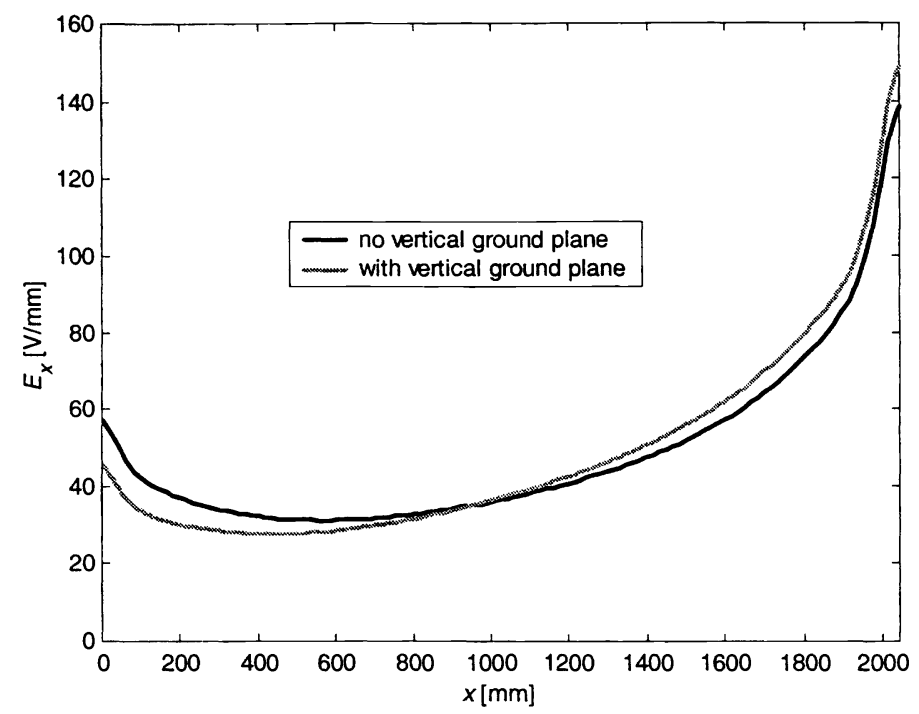

Figure 2. $E_{x}$ along the column axis for $100 \mathrm{kV}$ applied across the post insulator with and without a ground plane present.

Another approach that has been used in optical VTs is to integrate the field between two electrodes using a Pockels cell. The Pockels cell is placed in the space between the two electrodes with its ends in contact with them. In this case, the path of light traveling through the Pockels cell is also a path between the electrodes, typically parallel to a crystallographic axis of the Pockels cell, and is modulated by the component of the electric field that is parallel to that path. An example of this is described in Ref. 4, where an electro-optic crystal is used to integrate the field. Other examples of field integration based on piezoelectric fiber-optic sensors ${ }^{9}$ can also be found ${ }^{10,11}$.

For high-voltage applications, both controlling the geometry with specially shaped electrodes and exactly integrating the field with a Pockels cell have disadvantages. Special electrode structures for controlling the local geometry or for sandwiching a Pockels cell can result in high electric field stresses for high voltages. These high electric field stresses must be supported by special insulation, e.g., $\mathrm{SF}_{6}$ gas is often used for this purpose ${ }^{4}$.

\section{NUMERICAL INTEGRATION}

Here, an already existing insulating structure, specifically the post insulator introduced above, is transformed into a VT by strategically fitting it with only three small electric field sensors. The chosen number of sensors is determined by the desired accuracy of the voltage measurement and the most severe electric field distribution changes caused by the environment. The desired accuracy is related to the accuracy class of the sensor, e.g., metering or relaying classes. The most severe electric field perturbations due to the surrounding environment are dominated by surface contamination, worst-case weather conditions, and substation design standards, e.g., the clearances between conductors.

Since the voltage can be obtained by integrating the electric field, it can be approximated numerically, i.e., using a weighted sum. A numerical integration involves sampling the integrand, in this case the component of the electric field parallel to the path of integration, at a finite number of points, weighting the sampled values, and summing them. Hence, assuming the path of integration is the $x$-axis, one obtains

$$
\mathrm{V}_{b a}=-\int_{a}^{b} E_{x}(x) d x \approx-\sum_{i=1}^{N} \alpha_{i} E_{x}\left(x_{i}\right)
$$

where $E_{x}$ is the $x$-component of the electric field, $N$ is the number of samples, $\alpha_{i}$ is the weight on the $i$ th sample, and $x_{i}$ is the position of the $i$ th sample. The number of samples, and therefore the sample positions and weights, are chosen so that the weighted sum meets the accuracy requirements for all of the practically possible external influences. 
For $N$ electric field sensors, there are $2 N$ unknown variables, i.e., $N$ positions and $N$ weights. Ideally, these variables are chosen in such a way as to minimize the variation in the sum due to changes in the environment. Unfortunately, this is a formidable nonlinear minimization problem that becomes practically very difficult to solve due to the nature of the sum and the infinite number of possible external configurations (geometry and sources), particularly for $N>1$. Also, the importance of each configuration would have to be determined and taken into account in the minimization.

Instead of attempting to minimize this problem by brute force, it is pointed out that the $E_{x} s$ shown in Fig. 3 have a similar shape. $E_{x}$ can be written in terms of a kernel function $K(x)$ and a multiplicative scaling factor $\rho(x)$ :

$$
E_{x}(x)=\rho(x) K(x)
$$

Choosing $K$ to be the $E_{x}$ for the case of the post insulator with no external influences makes $\rho$ a nonuniform function for variations in the geometry (or a constant for the case of no geometric change). Figure 3 shows $\rho$ for the case of the vertical ground plane $1.5 \mathrm{~m}$ away from the post insulator. For this case and for practical geometric changes in general, $\rho$ is a smooth function. Thus, the shape of $\rho$ lends itself to being well-approximated by low order polynomials. Therefore, instead of minimizing the error in the sum with respect to an infinite number of configurations, the sum is minimized with respect to a few polynomials of ascending degree. Specifically, Eq. (1) is enforced for $\rho=1, x, x^{2}, \ldots, x^{2 N-1}$, giving $2 N$ equations and $2 N$ unknowns. Solving for $x_{i}$ and $\alpha_{i}$ gives a weighted sum that is exact for any $\rho$ that can be approximated by a polynomial of degree $2 N-1$ or less, which offers a trade-off between the expected severity of geometric changes and the number of sample points needed for a desired accuracy. In the following section, this method of measuring voltage is described in the design of a high-voltage IOVT.

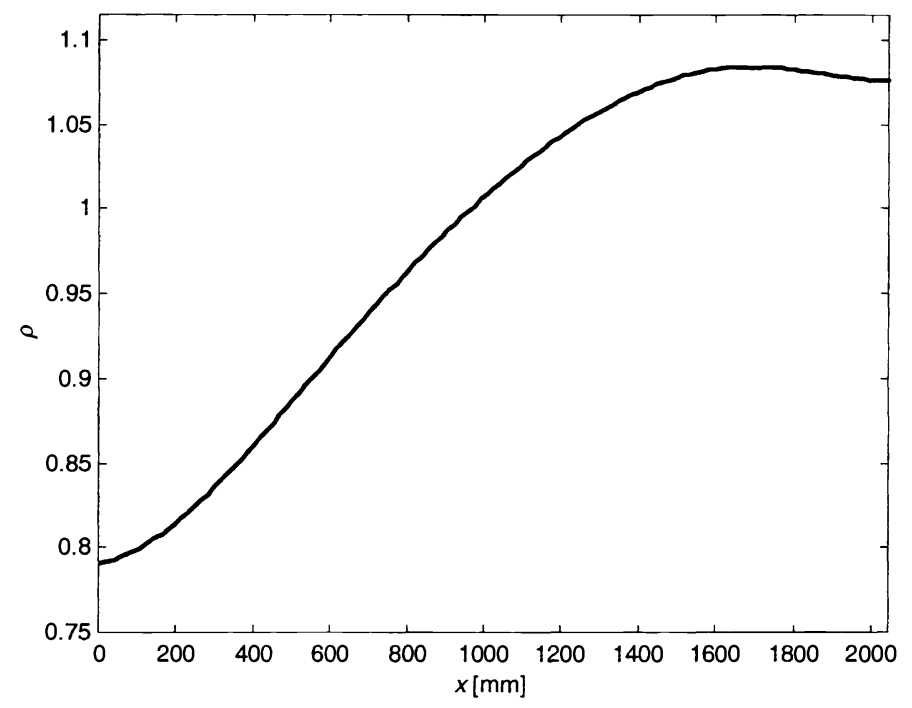

Figure 3. The scaling function $\rho$ representing the introduction of a vertical ground plane $1.5 \mathrm{~m}$ away from the post insulator.

\section{A HIGH-VOLTAGE INTEGRATED-OPTIC VOLTAGE TRANSDUCER}

A post insulator, like the one shown in Fig. 1(a) is transformed into a voltage transducer by mounting IOPC electric field sensors inside the inner, hollow, fiberglass tube. The positions and weights for the sensors are determined by $K$ and the number of sensors to be used. The number of sensors is predicated on the worst-case shape of $\rho$ that can occur in practice. The shape of $\rho$ is important because it determines the lowest degree of the approximating polynomial for which the numerical integration is exact. The field along the axis between the electrodes of the post insulator standing on a ground plane (as shown in Fig. 1(a)) is assigned to be the kernel function $K$. Here, the vertical ground plane case (see Fig. 1(b)) is representative of an overly extreme change of geometry, and the $\rho$ associated with it is used to determine $N$. Three sensors 
are chosen since $\rho$ (see Fig. 3) can be accurately approximated with a $5^{\text {th }}$ degree polynomial. Figure 4 shows the difference between $F$, the best (in the least-squares sense) $5^{\text {th }}$ degree polynomial fit to $\rho$, and $\rho$.

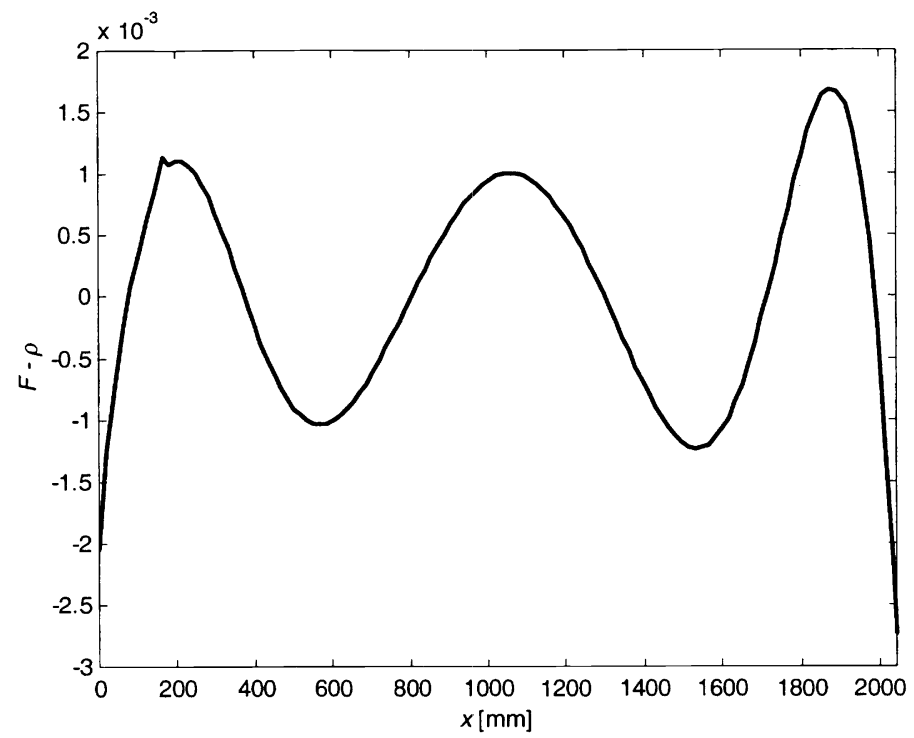

Figure 4. The difference between $F$, the best (in the least-squares sense) $5^{\text {th }}$ degree polynomial fit to $\rho$, and $\rho$.

Next, assuming that $\rho$ can be well-approximated by a $5^{\text {th }}$ degree polynomial, Eq. (2) is substituted into Eq. (1), and the following six equations in $x_{i}$ and $\alpha_{i}$ are set up (see Ref. 13):

$$
\begin{aligned}
& \int_{a}^{b} K(x) d x=\alpha_{1} K\left(x_{1}\right)+\alpha_{2} K\left(x_{2}\right)+\alpha_{3} K\left(x_{3}\right) \\
& \int_{a}^{b} K(x) x d x=\alpha_{1} K\left(x_{1}\right) x_{1}+\alpha_{2} K\left(x_{2}\right) x_{2}+\alpha_{3} K\left(x_{3}\right) x_{3} \\
& \int_{a}^{b} K(x) x^{2} d x=\alpha_{1} K\left(x_{1}\right) x_{1}^{2}+\alpha_{2} K\left(x_{2}\right) x_{2}^{2}+\alpha_{3} K\left(x_{3}\right) x_{3}^{2} \\
& \int_{a}^{b} K(x) x^{3} d x=\alpha_{1} K\left(x_{1}\right) x_{1}^{3}+\alpha_{2} K\left(x_{2}\right) x_{2}^{3}+\alpha_{3} K\left(x_{3}\right) x_{3}^{3} \\
& \int_{a}^{b} K(x) x^{4} d x=\alpha_{1} K\left(x_{1}\right) x_{1}^{4}+\alpha_{2} K\left(x_{2}\right) x_{2}^{4}+\alpha_{3} K\left(x_{3}\right) x_{3}^{4} \\
& \int_{a}^{b} K(x) x^{5} d x=\alpha_{1} K\left(x_{1}\right) x_{1}^{5}+\alpha_{2} K\left(x_{2}\right) x_{2}^{5}+\alpha_{3} K\left(x_{3}\right) x_{3}^{5}
\end{aligned}
$$

To solve this set of equations, the characteristic polynomial is constructed:

$$
P(x)=\left(x-x_{1}\right)\left(x-x_{2}\right)\left(x-x_{3}\right)=C_{0}+C_{1} x+C_{2} x^{2}+C_{3} x^{3},
$$

where $x_{1}, x_{2}$, and $x_{3}$ are both the sample positions and the roots of $P$, and $C_{0}, C_{1}, C_{2}$, and $C_{3}$ are the polynomial coefficients of $P$. The first equation in Eq. (3) is multiplied by $C_{0}$, the second by $C_{1}$, the third by $C_{2}$, and the fourth by $C_{3}$, and they are added together. Since $P$ is zero at its roots, the result is 


$$
C_{0} \int_{a}^{b} K(x) d x+C_{1} \int_{a}^{b} K(x) x d x+C_{2} \int_{a}^{b} K(x) x^{2} d x+C_{3} \int_{a}^{b} K(x) x^{3} d x=0
$$

This process is repeated starting with the second equation and then again with the third equation. Setting $C_{0}$ equal to 1 , the result is a linear system of equations:

$$
\left[\begin{array}{lll}
m_{1} & m_{2} & m_{3} \\
m_{2} & m_{3} & m_{4} \\
m_{3} & m_{4} & m_{5}
\end{array}\right]\left[\begin{array}{l}
C_{1} \\
C_{2} \\
C_{3}
\end{array}\right]=-\left[\begin{array}{l}
m_{0} \\
m_{1} \\
m_{2}
\end{array}\right]
$$

where

$$
m_{i}=\int_{a}^{b} K(x) x^{i} d x
$$

Solving this matrix equation gives the coefficients of $P$ and, then, finding the roots of $P$ gives the sample positions $x_{i}$. Finally, $\alpha_{1}, \alpha_{2}$, and $\alpha_{3}$ are computed by solving any three equations in Eq. (3), e.g.,

$$
\left[\begin{array}{l}
\alpha_{1} \\
\alpha_{2} \\
\alpha_{3}
\end{array}\right]=\left[\begin{array}{ccc}
K\left(x_{1}\right) & K\left(x_{2}\right) & K\left(x_{3}\right) \\
K\left(x_{1}\right) x_{1} & K\left(x_{2}\right) x_{2} & K\left(x_{3}\right) x_{3} \\
K\left(x_{1}\right) x_{1}^{2} & K\left(x_{2}\right) x_{2}^{2} & K\left(x_{3}\right) x_{3}^{2}
\end{array}\right]^{-1}\left[\begin{array}{l}
m_{0} \\
m_{1} \\
m_{2}
\end{array}\right]
$$

The resulting positions (with respect to point $a$ ) and weights (normalized to the weight of the middle sensor) of the sample points are given in Table 1. The condition given by Eq. (3) makes Eq. (1) exact for any $\rho$ that is a $5^{\text {th }}$-degree polynomial or less. A numerical integration of this type is known as a Gaussian quadrature ${ }^{12,13}$.

Table 1. Formula sample positions with respect to $a$ and weights normalized to the middle sensor's (Sample \#2) weight.

\begin{tabular}{|c|c|c|}
\hline Sample Number & $x_{i}[\mathrm{~mm}]$ & $\alpha_{i}$ \\
\hline 1 & 1868.8 & 0.4894 \\
\hline 2 & 1083.7 & 1.0000 \\
\hline 3 & 180.1 & 0.5944 \\
\hline
\end{tabular}

\section{SIMULATION AND TEST RESULTS}

The accuracy of the numerical integration was tested by using it to integrate $E_{x}$ for the simulated case of the post insulator with a vertical ground plane at a distance of $1.5 \mathrm{~m}$ (see Fig. 1(b)). Also, the percentage changes in the values of $E_{x}$ at the sample points, from those values of $E_{x}$ when there is no vertical ground plane present, are given in Table 2.

Using the principles described, an integrated-optic voltage transducer, with three immersion-type IOPCs mounted internally along the column axis, was constructed and tested in a high-voltage laboratory. The IOPCs were positioned at the sample point positions and were oriented so as to measure the vertical electric field component. Optical fibers, bonded directly to the IOPCs, transmitted light to and from the sensor-heads. The outputs of the sensors were weighted and summed by an A/D converter and a digital signal processor. The electronics had an absolute accuracy of $\pm 1 \%$. 
Table 2. Simulation percentage errors in voltage measurement $(\Delta V)$ and percentage changes in local electric field measurements $\left(\Delta E_{x}\left(x_{i}\right)\right)$ for a vertical ground plane 1.5 meters away.

\begin{tabular}{|c|c|}
\hline Variables & $\begin{array}{c}\text { \% Changes in } \\
\text { Values Due to a } \\
\text { Vertical Ground } \\
\text { Plane }\end{array}$ \\
\hline$\Delta V$ & -0.12 \\
\hline$\Delta E_{x}\left(x_{1}\right)$ & 8.1 \\
\hline$\Delta E_{x}\left(x_{2}\right)$ & 2.4 \\
\hline$\Delta E_{x}\left(x_{3}\right)$ & -18.7 \\
\hline
\end{tabular}

Introduction of the IOPCs and their holders along the column axis influence the field locally, but this effect is expected to introduce a very small error to the final measurement. This is due to the fact that the IOPC has dimensions on the order of millimeters (compared to the $2 \mathrm{~m}$ path length between electrodes) and each sensor holder is made of an insulating material with a low dielectric permittivity.

The IOVT was tested for accuracy for the case of a large, grounded screen standing vertically $1.5 \mathrm{~m}$ away from the center of the column. Water, ice, and any other deposits resting on the surface of the standoff sheds also constitute a change in the external geometry and may influence the electric field internal to the standoff. In particular, impure water or deposits that are conductive and that cover large regions of the shed surface may substantially affect $E_{x}$. Thus, the IOVT was also tested for accuracy using ANSI/IEEE and IEC wet testing specifications. The IOVT with the vertical grounded screen is shown in Fig. 5, and the lab test results are given in Table 3.

Table 3. Lab test percentage errors in voltage measurement $(\Delta V)$ and percentage changes in local electric field measurements $\left(\Delta E_{x}\left(x_{i}\right)\right)$ for several external conditions.

\begin{tabular}{|c|c|c|c|c|}
\hline Variables & $\begin{array}{c}\text { \% Changes in } \\
\text { Values Due to a } \\
\text { Vertical Grounded } \\
\text { Screen }\end{array}$ & $\begin{array}{c}\text { \% Changes in } \\
\text { Values for IEC Wet } \\
\text { Test Conditions }\end{array}$ & $\begin{array}{c}\text { \% Changes in } \\
\text { Values for ANSI } \\
\text { Wet Test Conditions }\end{array}$ & $\begin{array}{c}\text { \% Changes in } \\
\text { Values for Wet } \\
\text { Insulator Surface } \\
\text { with No Water Flow }\end{array}$ \\
\hline$\Delta V$ & 0.6 & 0.0 & -0.1 & 0.1 \\
\hline$\Delta E_{x}\left(x_{1}\right)$ & 19.1 & -0.1 & 0.4 & 0.6 \\
\hline$\Delta E_{x}\left(x_{2}\right)$ & -4.6 & 0.2 & 0.0 & 0.1 \\
\hline$\Delta E_{x}\left(x_{3}\right)$ & -22.9 & 0.1 & -0.4 & -0.3 \\
\hline
\end{tabular}

The changes in the $E_{x}\left(x_{i}\right)$ for the vertical ground plane simulation and for the vertical grounded screen lab test follow the same trend, i.e., the field increases near the top electrode and decreases near the bottom electrode. Differences between the simulated values and test values may be able to be attributed to the fact that the actual geometry of the test setup is not accounted for in the simulation, which only assumes that a semi-infinite ground plane is present. In contrast to the case of the vertical grounded screen, the water flow has a very small effect on the $E_{x}\left(x_{i}\right)$.

Voltage transducers for metering applications are required to meet accuracy class specifications from Class 1 to Class 0.2 . The corresponding magnitude error bands range from $\pm 1 \%$ to $\pm 0.2 \%$, respectively. Metering class specifications also impose limits on phase angle errors for each accuracy class. The voltage magnitude errors in Tables 2 and 3 show that the IOVT can meet Class 1 metering accuracy for magnitude and may, in practice, be able to meet Class 0.2 accuracy.

This VT provides a collection of advantages over previous approaches. It does not require special insulation since no particularly high electric field stresses are present. Its mechanical design is almost entirely that of the post insulator, which is available off-the-shelf. Primarily, the standoff serves to mechanically support the sensors and to protect them from harsh weather conditions. It also prevents electric field perturbations from occurring close to the sensors, which is important since such changes could introduce large errors into the integration. Here, IOPCs were used, but other small electric field sensors 
should also work. It should also be noted that the VT's standoff structure could also be placed between phases to give a measurement of the phase-to-phase voltage in a three-phase system.

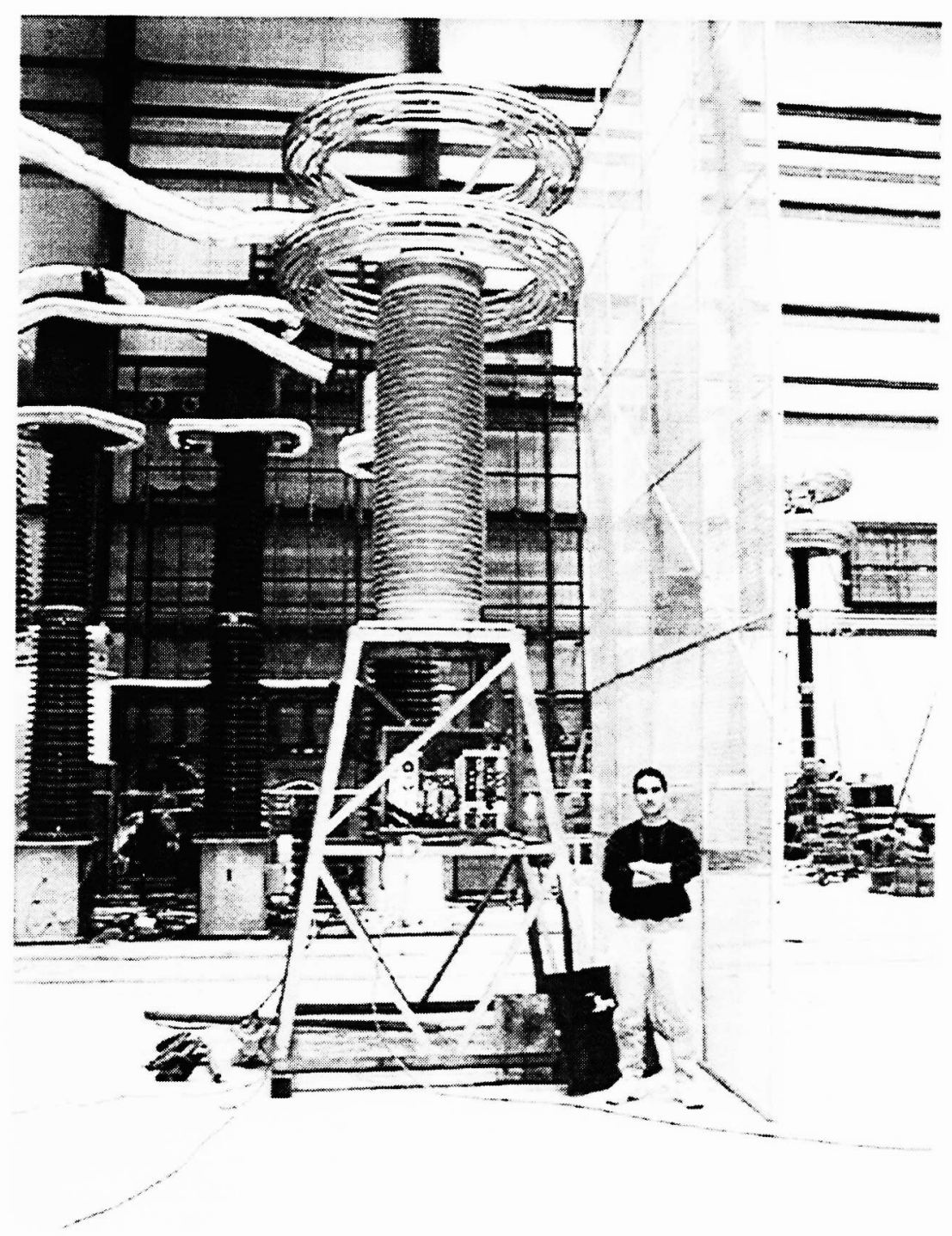

Figure 5. Integrated-optic voltage transducer with a vertical grounded screen $1.5 \mathrm{~m}$ away.

\section{SUMMARY AND CONCLUSION}

The concept of determining voltage using a very small number of electric field measurements and a customized numerical integration was introduced. A voltage transducer based on this concept was built. An IOVT, consisting of three IOPCs mounted inside a standard post insulator, was shown to be accurate even with significant changes in the electric field distribution. In conclusion, an IOPC-based voltage transducer can achieve Class 1 metering accuracy and may, in practice, be able to meet Class 0.2 accuracy. A key feature of this design concept is that it does not require special electrode structures and/or insulation, making it safe and environmentally friendly. 


\section{REFERENCES}

1. A. Bosco, T. Hertig, and A. Kaczkowski, "Measuring device for a metal-enclosed, gas-insulated high-voltage installation," US Patent 5,917,316, June 29, 1999.

2. R. Baumgartner, K. Y. Haffner, H. Hageli, and A. Kaczkowski, "Current and voltage transformer for a metal encapsulated, gas-insulated high-voltage installation," US Patent 5,272,460, December 21, 1993.

3. L. H. Christensen, "Design, construction, and test of a passive optical prototype high voltage instrument transformer," IEEE Transactions on Power Delivery, Vol. 10, No. 3, pp. 1332-1337, July 1995.

4. S. Weikel and G. Stranovsky, "Application of an electro optic voltage transducer at $345 \mathrm{kV}$," EPRI Optical Sensors for Utility T\&D Applications Workshop, Portland, Oregon, July 20-21, 1995.

5. N. A. F. Jaeger and F. Rahmatian, "Integrated optics pockels cell high-voltage sensor," IEEE Transactions on Power Delivery, Vol. 10, No. 1, pp. 127-134, January 1995.

6. S. V. Marchall and G. G. Skitek, Electromagnetic Concepts and Applications, Prentice-Hall, London, 1990.

7. O. C. Zienkiewicz, The Finite Element Method, McGraw-Hill, London, 1977.

8. B. H. McDonald and A. Wexler, "Finite-element solution of unbounded field problems," IEEE Transactions on Microwave Theory and Techniques, Vol. 20, No. 12, pp. 841-847, December 1972.

9. K. M. Bohnert and J. Nehring, "Fiber-optic sensing of electric field components," Applied Optics, Vol. 27, No. 23, pp. 4814-4818, December 1988.

10. K. Bohnert and J. Nehring, "Fiber-optic sensing of voltages by line integration of the electric field," Optics Letters, Vol. 14, No. 5, pp. 290-292, March 1989.

11. M. Bjarme and I. Bassett, "High Voltage Measuring Device," International Patent Application, Publication No. WO 97/01100, January 9, 1997.

12. C. E. Froberg, Introduction to Numerical Analysis, Reading, MA: Addison-Wesley, pp. 172-201, 1965.

13. R. W. Hamming, Numerical Methods for Scientists and Engineers, McGraw-Hill, New York, pp. 130-132, 1962. 